\title{
Measuring Helical FCG Voltage with an Electric Field Antenna
}

A. D. White, R. A. Anderson, J. B. Javedani, D. B. Reisman, D. A. Goerz, A. J. Ferriera, R. D. Speer

August 4, 2011

18th IEEE International Pulsed Power Conference Chicago, IL, United States June 19, 2011 through June 23, 2011 
This document was prepared as an account of work sponsored by an agency of the United States government. Neither the United States government nor Lawrence Livermore National Security, LLC, nor any of their employees makes any warranty, expressed or implied, or assumes any legal liability or responsibility for the accuracy, completeness, or usefulness of any information, apparatus, product, or process disclosed, or represents that its use would not infringe privately owned rights. Reference herein to any specific commercial product, process, or service by trade name, trademark, manufacturer, or otherwise does not necessarily constitute or imply its endorsement, recommendation, or favoring by the United States government or Lawrence Livermore National Security, LLC. The views and opinions of authors expressed herein do not necessarily state or reflect those of the United States government or Lawrence Livermore National Security, LLC, and shall not be used for advertising or product endorsement purposes. 


\title{
Measuring Helical FCG Voltage with an Electric Field Antenna*
}

\author{
A.D. White, R. A. Anderson, J. B. Javedani, D. B. Reisman, D.A. Goerz A. J. Ferriera, \\ R. D. Speer \\ Lawrence Livermore National Laboratory, PO Box 808, L-153 \\ Livermore, CA 94550, USA
}

\begin{abstract}
A method of measuring the voltage produced by a helical explosive flux compression generator using a remote electric field antenna is described in detail. The diagnostic has been successfully implemented on several experiments. Measured data from the diagnostic compare favorably with voltages predicted using the code CAGEN [1], validating our predictive modeling tools. The measured data is important to understanding generator performance, and is measured with a low-risk, minimally intrusive approach.
\end{abstract}

\section{INTRODUCTION}

We have designed and tested several explosive flux compression generators within the last decade. Thorough descriptions of the design and performance of these generators have been published elsewhere [2] [3] [4], and are beyond the scope of this paper. To briefly summarize these results, we have used a helical generator of our design in series with a coaxial generator of our design to deliver tens of megaamperes and tens of megajoules to low inductance loads.

A drawing of one such generator, the Mini-G, is shown in Fig. 1. A photograph of a Mini-G on the shot table is shown in Fig. 2, and the current waveform from Mini-G Test 2 (MGT2) is shown in Fig. 3.

Several years ago, during one such experiment, we observed an anomaly in the data from diagnostics that were external to the generator. Analysis of this anomaly suggested that 1-2 kiloamperes of current were driven into an external ground loop, although operation of the generator was nominal and unaffected. After further analysis of the external generator circuit, we determined that the shield and inner conductor of the seed capacitor bank cables were becoming tied together, and tied to the top of the helical coil through a low-impedance connection formed by the crowbar of the moving helical generator armature. The floating capacitor bank would

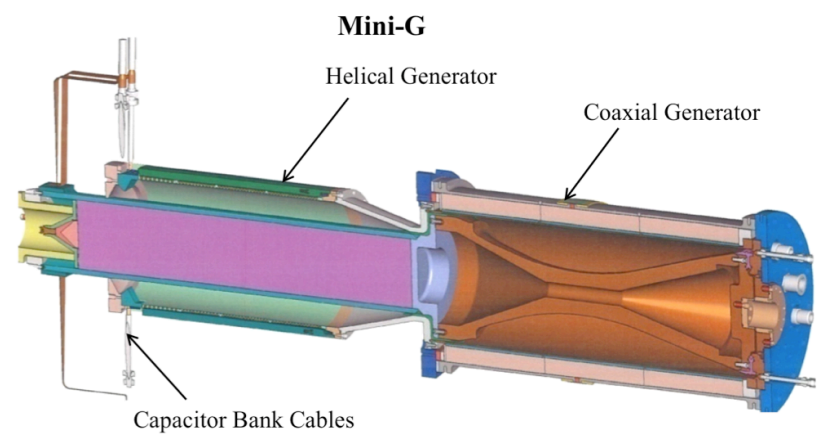

Figure 1. Drawing of the Mini-G. The helical generator consists of a copper helical coil stator and an aluminum cylindrical armature. HE initiation occurs at a singlepoint at the top of the helical generator. The coaxial generator is imploded via 51 detonators that are uniformly spaced around the waist of the coaxial generator.

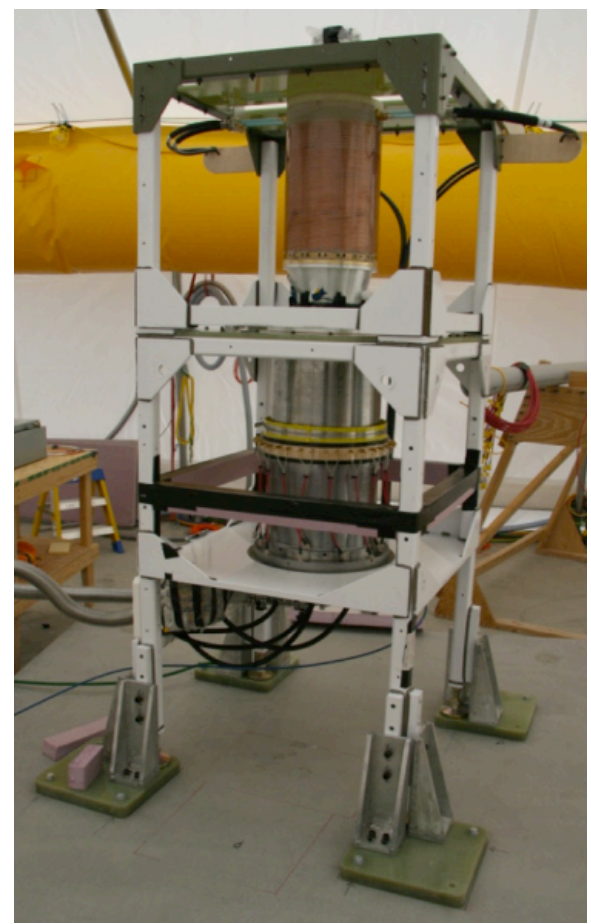

Figure 2. A photograph of a Mini-G on the firing table

\footnotetext{
* This work performed under the auspices of the U.S. Department of Energy by Lawrence Livermore National Laboratory under Contract DE-AC52-07NA27344.
} 
therefore be lifted to the potential of the top of the helical coil relative to both the grounded tamper of the coaxial generator, and the grounded enclosure that the capacitor bank is located within. A voltage breakdown between the floating capacitor bank and the grounded enclosure combined with our estimates of the external circuit impedance explained the 1-2 kA external ground fault.

Computational modeling of the helical generator using CAGEN predicted a coil voltage of around $-40 \mathrm{kV}$, and high-potting shortly after the shot where the suspected breakdown occurred failed at around $-37.5 \mathrm{kV}$ - slightly less than the predicted voltage.

On subsequent shots, we placed an electric field antenna in the vicinity of the capacitor bank, to both verify the capacitor bank did indeed elevate to the potential of the top of the coil, and determine if we could measure the coil voltage using a remote electric field antenna. A sketch of the complete external circuit is shown in Fig. 4.

The remainder of this paper describes the details concerning this measurement, presents measured data from several experiments, and discusses comparisons between the measured and modeled voltages.

\section{ANTENNA DESIGN AND IMPLEMENTATION}

Numerous diagnostics could be used to measure the voltage that the capacitor bank is elevated to during experiment. The requirements were straightforward - the diagnostic should be low-risk, and not inadvertently cause or contribute to a breakdown; the diagnostic should produce a signal in the hundreds of millivolts, to make use of existing fiber optic link hardware that was available; and the diagnostic should have a simple transfer function that is measurable in the bandwidth of interest through an in situ calibration.

An electric field monopole antenna was chosen. It is low-risk because it is non-contact and can be located away from the high potential of the capacitor bank. The length of the monopole element and the addition of shunt capacitance can be used to control the two most important parameters of the antenna response: the amplitude of the response and the droop. In addition, such antennas are simple to make and robust, and their performance is well understood from first principles.

Electrostatic modeling of the inside of the seed bank enclosure was used as a tool to help determine placement of the antenna. An appropriate location would have both a sufficiently large electric field along the antenna that a suitable signal level would be produced, but also be in a location that was fairly insensitive to antenna movement, so that small movement of the antenna from shot to shot would not strongly affect the antenna's in situ sensitivity. Further, the antenna needed to be located in a place that

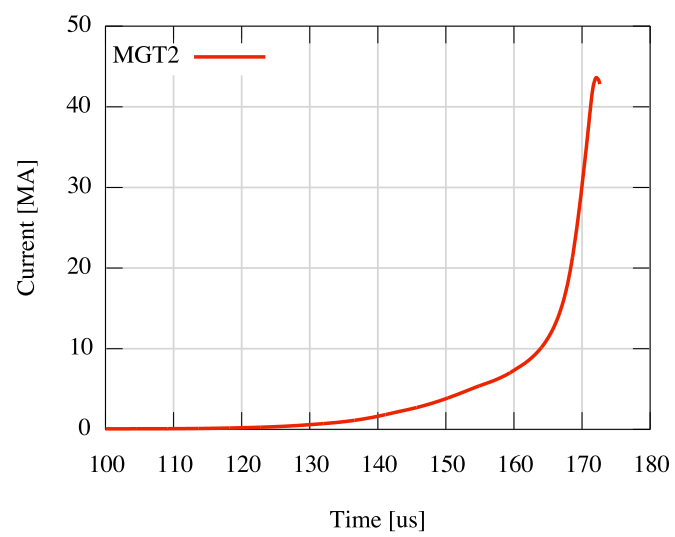

Figure 3. The current waveform from Mini-G Test 2

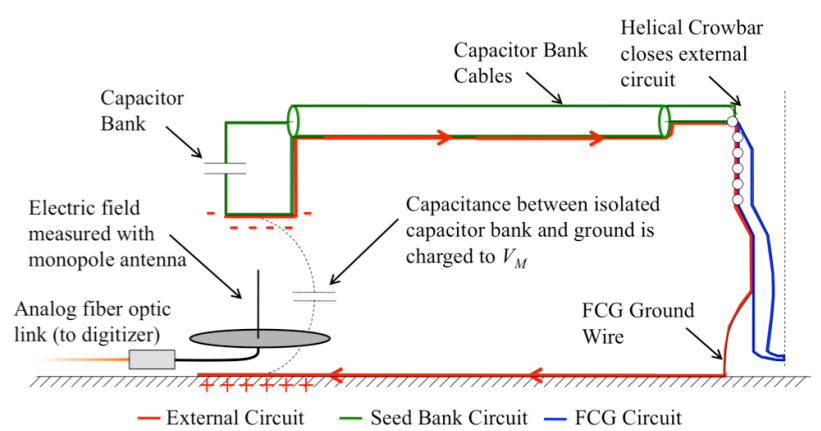

Figure 4. A circuit sketch of the measurement. After helical generator crowbar, the experiment consists of three coupled circuits - a seed bank circuit, an internal FCG circuit, and an external circuit. In the absence of faults, the current in the seed bank circuit is on the order of $\mathrm{kA}$, the current in the external circuit is on the order of $\mathrm{A}$, and the current in the internal FCG circuit is on the order of MA (see Fig. 3).

would not present a significant obstacle to personnel maintaining and operating the capacitor bank in the cramped enclosure.

An excerpt from the electrostatic modeling, and the approximate final position of the antenna is shown in Fig. 5.

The antenna was built using a 24" diameter ground plane, and a monopole element of variable length. The final length of the monopole element was determined during in situ calibration. Prior to installation, the antenna's frequency response was measured in a TEM cell. These measurements showed that the antenna response had a lower $3 \mathrm{~dB}$ point of around $1 \mathrm{kHz}$ when the antenna was terminated with a $5 \mathrm{~ns}$ cable and the analog fiber optic link with no shunt capacitance. Adding the shunt capacitance we expected would be necessary (4.5 $\mathrm{nF}$ or $9 \mathrm{nF}$, depending on the generator) to attenuate the signal to an appropriate level lowered the $3 \mathrm{~dB}$ point to under $100 \mathrm{~Hz}$. The antenna response is therefore essentially flat during the timescale of interest, which is on the order of tens of microseconds. 

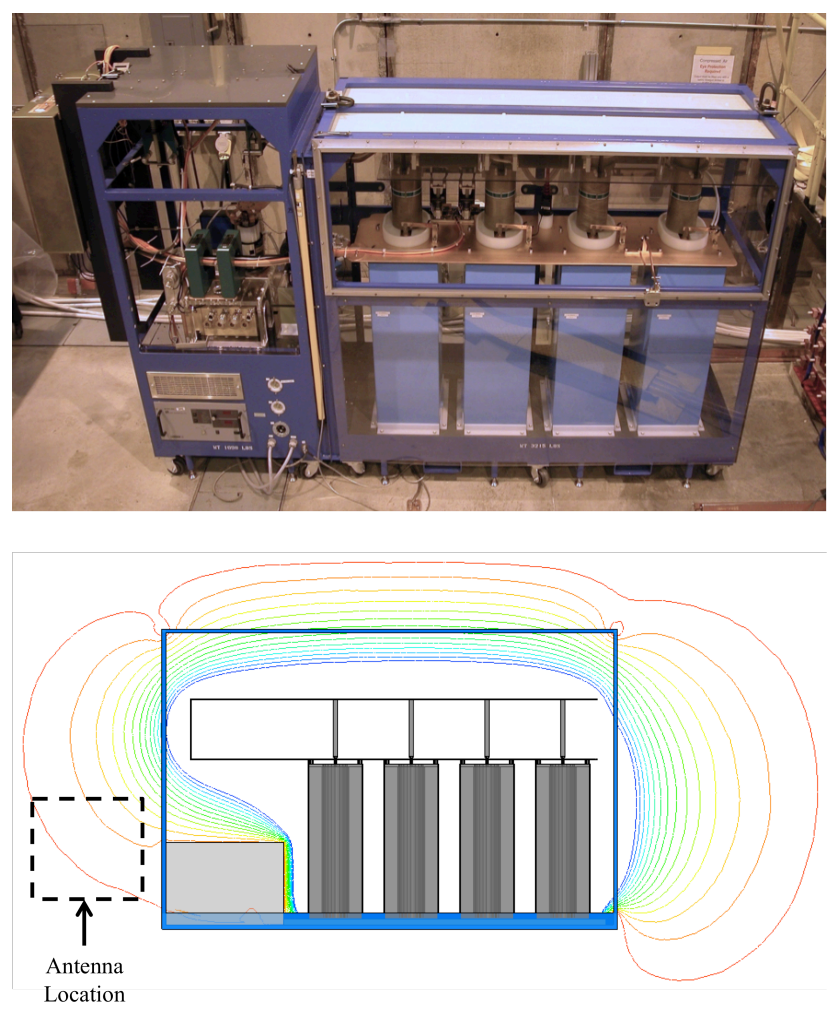

Figure 5. A photograph of the capacitor bank prior to installation in the enclosure (top). An excerpt from the electrostatic field modeling of the inside of the capacitor bank enclosure. Equipotential contours are shown. The approximate position of the antenna is indicated by the dashed box (bottom).

The antenna was installed in the location suggested by the electrostatic modeling. The output of the antenna was connected to an analog fiber optic link, which converts the measured voltage into an optical signal. This signal is routed via optical fiber to recording digitizers located within the main bunker. Figure 6 is a photo showing the antenna in the installed location.

An in situ calibration was performed to measure the in situ antenna sensitivity. The calibration was straightforward: the capacitor bank was isolated from ground as it is during the shot, and a CDU was discharged between the grounded enclosure and the capacitor bank cable shields. This applied a voltage in the same location as the helical coil applies a voltage during the experiment. The applied voltage was measured, and compared with the voltage produced by the antenna. The shunt capacitance and antenna monopole element length were varied until the antenna sensitivity was at the desired value. A sketch of the in situ calibration is shown in Fig. 7.

\section{A COMPARISON BETWEEN MEASURED AND COMPUTED RESULTS}

The antenna was used to measure helical coil voltages on several experiments during the last two years.

The results confirmed our expectation - that the floating capacitor bank is lifted in potential by tens of $\mathrm{kV}$ during experiment.

In order to make meaningful comparison with CAGEN the differences between what is modeled with CAGEN, and what is measured during experiment need to be examined.

\section{A. Measured quantities}

The calibrated electric field antenna measures the difference in potential between the low side of the floating capacitor bank and the ground plane. The current flowing in the external circuit (see Fig. 4) is small, so applying Kirchoff's voltage law to the external circuit reveals only three terms: 1) the measured voltage between the floating capacitor bank and the ground plane, 2) the voltage developed along the helical generator coil, and 3) the voltage developed along the shield of the capacitor bank cables from the seed bank current.

Writing out Kirchhoff's voltage law for the loop, the measured voltage can be described by:

$$
V_{M}=-I_{S B} Z_{T}+V_{C o i l}
$$

where $V_{M}$ is the measured voltage, $I_{S B}$ is the current flowing on the inside of the seed bank cables, $Z_{T}$ is the effective transfer impedance of the capacitor bank cables (approximated by the DC resistance of the shield of the four seed bank cables in parallel, which is approximately 20 milliohms), and $V_{\text {Coil }}$ is the voltage developed across the coil.

$V_{\text {Coil }}$ can further be described by:

$$
V_{\text {Coil }}=\dot{\phi}_{H G}+I_{G} R_{\text {Coil }}
$$

where $\phi_{H G}$ denotes the magnetic flux linking the coil within the helical generator, the diacritical dot denotes time derivative, $I_{G}$ denotes the current flowing in the flux compression generator, and $R_{\text {Coil }}$ denotes the resistance of coil. 


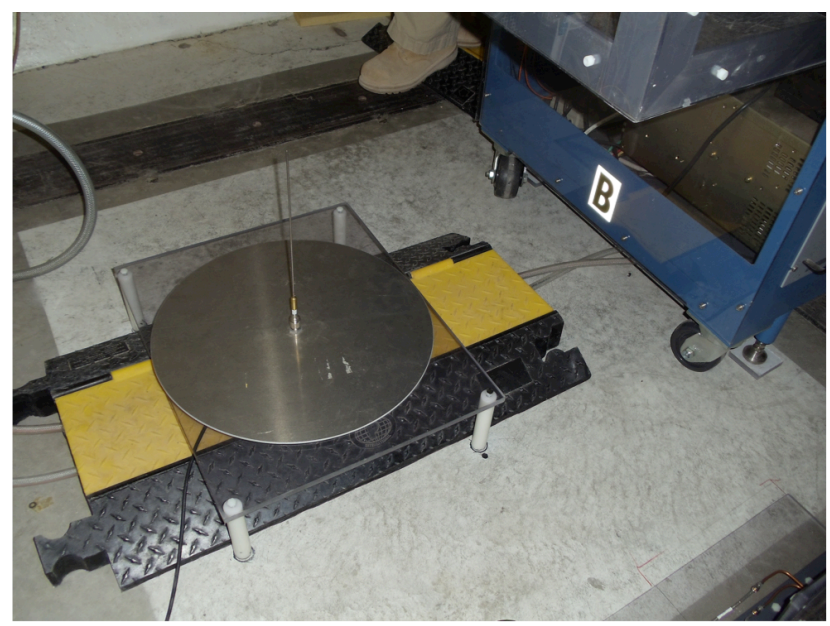

Figure 6. The installed antenna. The capacitor bank chassis is the blue frame in the upper-right portion of the photo. The electric field antenna has a ground plane that is 24 " in diameter, and a monopole element that is 16 " in height. The shunt capacitor is connected directly to the antenna output, below the ground plane. The cable visible in the lower left of the photograph is connected to an analog fiber optic link.

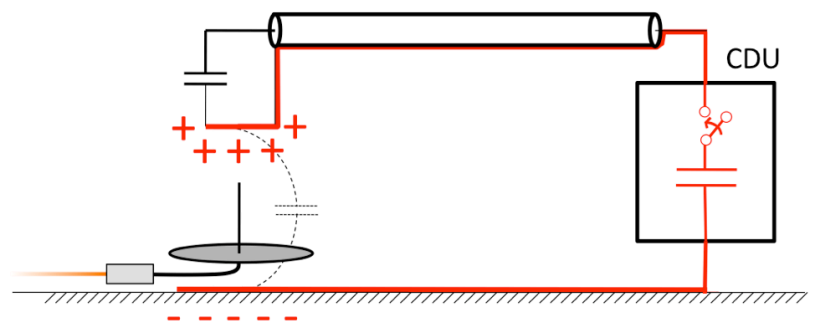

Figure 7. A sketch of the in situ calibration circuit. A $500 \mathrm{nF}$ CDU is charged to $4 \mathrm{kV}$ and discharged between the shield of the capacitor bank cables and the ground plane. Not shown is a $200 \mathrm{Ohm}$ shunt resistor across the CDU output which provides damping of the response. Both the CDU output and antenna output are exponential tail pulses with a time constant of approximately 100 us. The ratio between the measured antenna voltage and the applied voltage is the antenna sensitivity.

Fig. 8 shows the three terms in Eq. 1 from Mini-G Test 2 , where $I_{S B}$ is the seed bank current measured during experiment, $V_{M}$ is the antenna voltage measured during experiment, and $Z_{T}$ is the DC resistance of the cable shields, measured prior to experiment.

\section{B. Modeled quantities}

The computational tool CAGEN computes the voltage at the output of the helical generator, which is:

$$
V_{H G}=\dot{\phi}+I_{G} R_{H G}
$$

where $V_{H G}$ is the voltage produced at the helical generator output, and $R_{H G}$ is the total resistance of the helical generator, equal to

$$
R_{H G}=R_{\text {Coil }}+R_{\text {Armature }}
$$

where $R_{\text {Armature }}$ is the resistance of the aluminum armature. $V_{\text {Coil }}$ - the voltage between the top and bottom of the coil-can be computed from CAGEN by subtracting the voltage drop along the armature from Eq. 3. The result is identical to Eq. 2:

$$
V_{\text {Coil }}=V_{H G}-I_{G} R_{\text {Armature }}=\dot{\phi}_{H G}+I_{G} R_{\text {Coil }}
$$

\section{Measurement and Modeling results}

The measured and modeled values for $V_{\text {Coil }}$ from MiniG Test 2 are shown in Fig. 9. Two CAGEN waveforms are shown, due to known discrepancies between the CAGEN predictions and experiment. CAGEN1 is the computed $V_{\text {Coil }}$ using a seed current that is known to be incorrect, but this CAGEN model accurately predicts current out of the helical generator during helical generator performance. CAGEN2 is the computed $V_{\text {Coil }}$ using a seed current that matches experiment, but this CAGEN model over-predicts current out of the helical generator during helical generator performance by around $26 \%$. We are working with the CAGEN developers to better understand these discrepancies.

A method of verifying our understanding of the external circuit is possible by measuring the external circuit current. The integrated external circuit current scaled by the capacitance between the isolated capacitor bank and the ground plane should have the same waveform shape and amplitude as the voltage measured from the electric field antenna. This comparison, using data from Mini-G Test 4, is shown in Fig. 10. The capacitance used for scaling was inferred by scaling the integrated current data until amplitude agreement was observed. This resulted in a capacitance of $1.8 \mathrm{nF}$, which is in reasonable agreement with the measured value of around $1.9 \mathrm{nF}$, which varied somewhat depending on the technique used. There is good agreement between the two waveforms during the entire time record.

\section{Summary}

A method of measuring the voltage developed across the coil of a helical explosive flux compression generator is described. The approach is non-intrusive and low-risk, and provides a means of diagnosing internal generator fields from a distance. The measurement has been successfully implemented on several experiments. The results have been repeatable, and consistently agree well with modeling performed using the code CAGEN. 


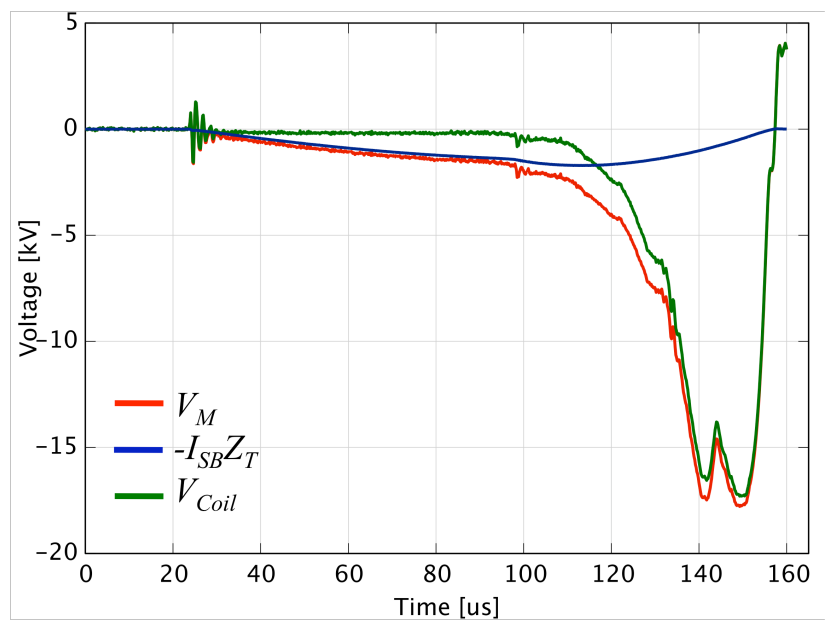

Figure 8. Overlay of terms from Eq. 1, measured during Mini-G Test 2. The capacitor bank discharges at approximately 24 us; helical generator crowbar occurs at approximately 95 us. The initial transient rings at approximately 25 us occurs when the seed capacitor bank discharges, and rings at the frequency of oscillation of the external circuit, which is approximately $700 \mathrm{kHz}$. The sign on the measured voltage is negative, which implies the top of the coil attains a negative voltage relative to the bottom.

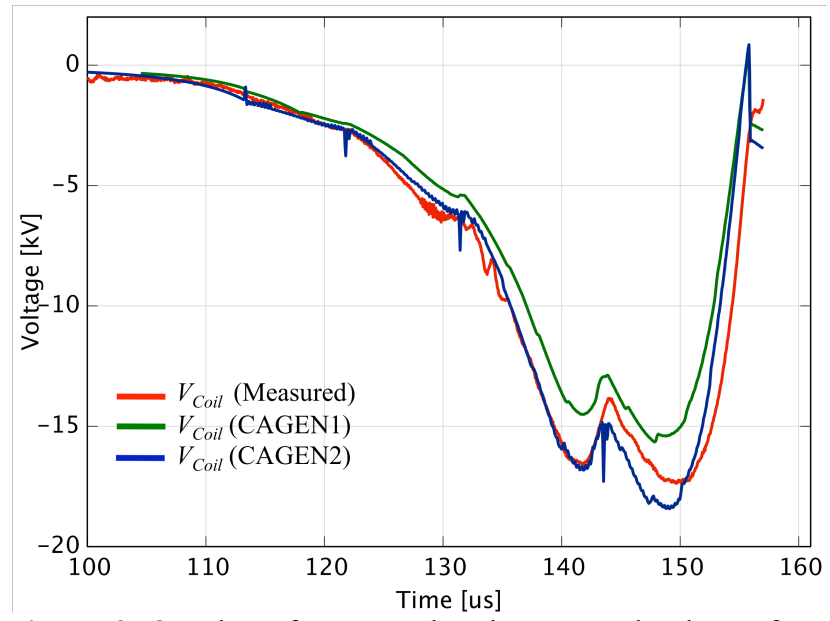

Figure 9. Overlay of measured and computed values of $V_{\text {Coil }}$ from Mini-G Test 2.

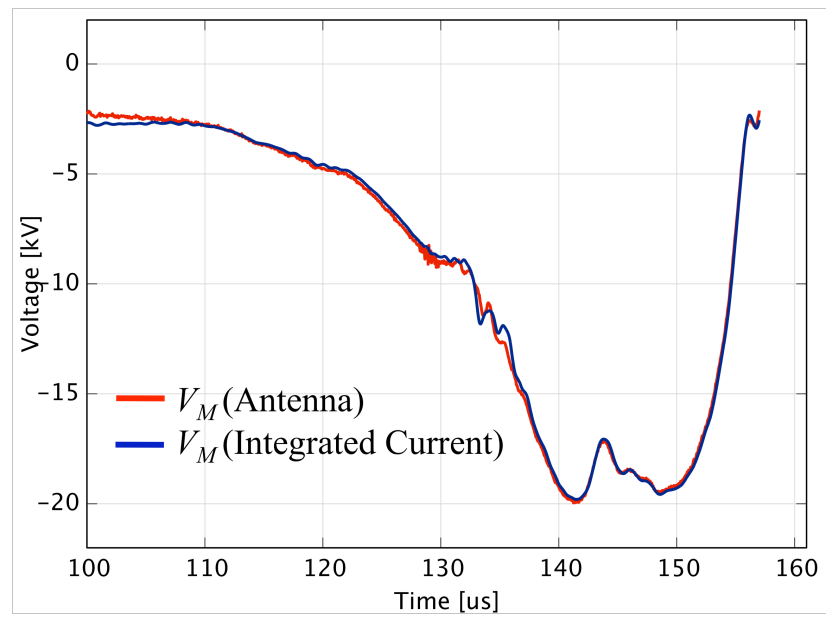

Figure 10. The measured voltage from the antenna compared with the integrated external circuit current

\section{REFERENCES}

[1] J. B. Chase, D. Chato, G. Peterson, P. Pincosy, and G. F. Kiuttu, "CAGEN: A modern PC based computer modeling tool for explosive MCG generators and attached loads", Proc. $12^{\text {th }}$ IEEE International Pulsed Power Conference, 1999, pp. 597-600.

[2] D. B. Reisman et al., "The advanced helical generator," Review of Scientific Instruments, vol. 81, 2010 .

[3] D. B. Reisman et al., "The full function test explosive generator," Review of Scientific Instruments, vol. 81, 2010 .

[4] D. B. Reisman, "Mini-G: the development of an optimized FCG device", Proc. $18^{\text {th }}$ IEEE

International Pulsed Power Conference, 2011, these proceeedings 